Bull. Austral. Math. Soc.

VOL. 64 (2001) [315-320]

\title{
COPRIME ACTIONS AND DEGREES OF PRIMITIVE INDUCERS OF INVARIANT CHARACTERS
}

\author{
Alexander Moretó and Lucía Sanus
}

\begin{abstract}
Let a finite group $A$ act coprimely on a finite group $G$ and $\chi \in \operatorname{Irr}_{A}(G)$. Isaacs, Lewis and Navarro proved that if $G$ is nilpotent then the degrees of any two $A$-primitive characters of $A$-invariant subgroups of $G$ inducing $\chi$ coincide. In this note we aim at extending this result by weakening the hypothesis on $G$.
\end{abstract}

\section{INTRODUCTION}

Let $A$ and $G$ be finite groups of coprime order. If $A$ acts on $G$, we write $\operatorname{Irr}_{A}(G)$ to denote the set of $A$-invariant irreducible characters of $G$. This set of characters has been widely studied. Also, the $A$-version of several usual character theoretic concepts has been defined. For instance, recall that an $A$-invariant character $\chi \in \operatorname{Irr}(G)$ is $A$-primitive if it is not induced from any $A$-invariant character of any $A$-invariant proper subgroup. We say that $\chi$ is $A$-monomial if it is induced from an $A$-invariant linear character of an $A$-invariant subgroup. Isaacs, Lewis and Navarro have recently proved the following result $[4$, Theorem $\mathrm{B}]$, which we state for the ease of reference.

THEOREM 1.1. Let $A$ act coprimely on a finite nilpotent group $G$ and $\chi \in$ $\operatorname{Irr}_{A}(G)$. Then the degrees of any two $A$-primitive characters of $A$-invariant subgroups inducing $\chi$ coincide.

Navarro has asked to what extent it is possible to weaken the nilpotency hypothesis and, in particular, whether the result holds when $G$ is supersolvable. In this note we show that Theorem 1.1 cannot be extended to supersolvable groups, but that it is possible to generalise it along other directions.

THEOREM A. There exists a supersolvable group $G$ acted on by a cyclic group $A$ with an $A$-monomial irreducible character $\chi$ which is also induced from some non-linear A-primitive character.

Received 28th February, 2001

The first author was supported by the Basque Government and the University of the Basque Country UPV 127.310-EB160/98. The second author was partially supported by DGICYT.

We are very much indebted to $M$. Isaacs and $G$. Navarro for many fruitful discussions. The work of the first author was done while he was visiting the University of Valencia and part of that of the second one while she was visiting the University of Wisconsin-Madison. We would like to take this opportunity to thank both Mathematics Departments for their hospitality.

Copyright Clearance Centre, Inc. Serial-fee code: 0004-9727/01 \$A2.00+0.00. 
The degree of the irreducible character $\chi$ of the group constructed in Theorem A is not a prime power. As in a number of situations when dealing with induction of characters, characters of prime power degree also have a different behaviour in this problem (examples of this different behaviour are, for instance, Theorem $A$ of $[\mathbf{4}],[\mathbf{1}]$ and $[\mathbf{6}]$ ). In fact, for characters of prime power degree it is possible to replace the supersolvability assumption by a much weaker one, namely that the group $G$ is metanilpotent, in order to obtain the desired equal-degrees conclusion.

Theorem B Let $A$ act coprimely on a supersolvable group $G$ and assume that $\chi \in \operatorname{Irr}_{A}(G)$ is such that $\chi(1)$ is a prime power. Then the degrees of any two A-primitive characters inducing $\chi$ coincide.

Theorem A shows that it is not possible to extend Theorem 1.1 to nilpotent-byAbelian $M$-groups. However, we are able to extend it to Abelian-by-nilpotent groups. In fact, we prove more.

Theorem C Let $A$ act coprimely on a solvable group $G$ and let $\chi \in \operatorname{Irr}_{A}(G)$. Assume that there exists a normal subgroup $N$ of $G$ such that $G / N$ is nilpotent and $N$ has Abelian Sylow subgroups. Then the degrees of any two A-primitive irreducible characters inducing $\chi$ coincide.

\section{Proof of Theorem A}

We begin with the proof of Theorem $A$.

THEOREM 2.1. There exists a supersolvable group $G$ acted on by a cyclic group $A$ with an $A$-monomial character which is induced from an $A$-primitive irreducible character of degree 25.

Proof: Let $P$ be the extraspecial 5-group of order $5^{5}$ and exponent 5, that is, $P$ has generators $x, y, u, v$ and $z$ all of them of order 5 and the following non-trivial relations:

$$
[x, y]=[u, v]=z
$$

and let $Q$ be the dihedral group

$$
Q=\left\langle g, h \mid g^{2}=1=h^{4}, h^{g}=h^{-1}\right\rangle .
$$

Now, let $G=P Q$ where $Q$ acts on $P$ with the following nontrivial relations:

$$
u^{g}=u^{-1}, v^{g}=v^{-1}, x^{h}=x^{-1}, y^{h}=y^{-1}, u^{h}=u^{-1}, v^{h}=v^{-1} .
$$

We have that $G$ is supersolvable and $Z(G)=\left\langle z, h^{2}\right\rangle$. Now, let $A=\langle a\rangle$ be the cyclic group of order 3 acting trivially on $Q$ and with the following nontrivial relations on $P$ :

$$
x^{a}=y, y^{a}=x^{-1} y^{-1}, u^{a}=v, v^{a}=u^{-1} v^{-1} .
$$


It is a routine to check that the action of $A$ on $G$ is well-defined. Now, consider $X=$ $\left\langle x u^{2}, y v^{2}, z\right\rangle \leqslant P$ and put $Y=\left\langle x u^{2}, y v^{2}\right\rangle$. Since $P$ has nilpotency class 2 ,

$$
\left[x u^{2}, y v^{2}\right]=[x, y]\left[u^{2}, v^{2}\right]=z z^{4}=1
$$

Hence $X$ is Abelian. Write $H=\langle h\rangle$. We have that $X$ is an $A H$-invariant normal subgroup of $P$. Since $X$ is $H$-invariant, $V=X H$ is a subgroup of $G$ of index 50. Let $\delta$ be a non-trivial irreducible character of $Z(P)$. Let $\lambda=\delta \times 1_{Y} \in \operatorname{Irr}_{A}(X)$ which is also $H$-invariant. Let $\hat{\lambda}$ be the canonical extension of $\lambda$ to $V A$ (see [3, Lemma 13.3]) and $\tilde{\lambda}=\hat{\lambda}_{V} \in \operatorname{Irr}_{A}(V)$, which is the canonical extension of $\lambda$ to $V$. Now, consider a linear character $\mu$ of $H$ such that $\mu_{Z(Q)} \neq 1_{Z(Q)}$. Let $\widehat{\mu}$ be the extension of $\mu$ to $P H$ such that $P \leqslant \operatorname{Ker} \hat{\mu}$ and $\tilde{\mu}=\widehat{\mu}_{V}$, which is the extension of $\mu$ to $V$ such that $X \leqslant \operatorname{Ker} \tilde{\mu}$. Define $\beta=\tilde{\lambda} \tilde{\mu} \in \operatorname{Irr}_{A}(V)$.

We claim that there exists an $A$-invariant linear character of $V$ which induces irreducibly to $G$. Since $\lambda$ induces irreducibly to $P, \tilde{\lambda}^{P H} \in \operatorname{Irr}(P H)$. We may assume that $\tilde{\lambda}^{P H}$ is $G$-invariant (otherwise, $\tilde{\lambda}^{G}$ would be irreducible and the claim would follow). We have that

$$
\beta^{P H}=(\tilde{\lambda} \widetilde{\mu})^{P H}=\tilde{\lambda}^{P H} \widehat{\mu} \in \operatorname{Irr}(P H),
$$

by $[3$, Problem 5.3]. Since $\mu$ is not $Q$-invariant (because $Z(Q) \notin \operatorname{Ker} \mu$ ), $\hat{\mu}$ is not $\dot{G}$ invariant, and thus $\beta^{P H}$ is not $G$-invariant. Consequently, $\beta^{G} \in \operatorname{Irr}_{A}(G)$ and the claim holds.

Let $\chi \in \operatorname{Irr}_{A}(G)$ be induced from an $A$-invariant linear character of $V$. Set $U=$ $\left\langle g, h^{2}\right\rangle[P]$. We claim that $50 \notin \mathrm{cd}(U)$. Otherwise, let $\psi \in \operatorname{Irr}(U)$ such that $\psi(1)=50$. By [3, Corollary 11.29], $\psi$ lies over an irreducible character $\phi$ of $P$ such that $\phi(1)=25$. Now, $\phi$ is fully ramified with respect to $Z(P)$. Since $Z(P)$ is central in $G, \phi$ is $Q$-invariant. By [3, Problem 6.18], there exists $\tilde{\phi}$ extension of $\phi$ to $U$ and, by Gallagher's theorem [3, Corollary 6.17]), the irreducible characters of $U$ lying over $\phi$ are $\tilde{\phi} \tau$ where $\tau \in \operatorname{Irr}(U / P)$. Now the contradiction follows from the fact that $U / P$ is Abelian. We deduce that there exists $\psi \in \operatorname{Irr}_{A}(U)$ lying under $\chi$ such that $\psi(\mathbf{1})=25$ (we are using [3, Theorem 13.27]).

It is clear that $\psi^{G}=\chi$ and to conclude the proof it is enough to show that $\psi$ is $A$ primitive. We argue by the way of contradiction. Let $K$ be a proper $A$-invariant subgroup of $U$ with an $A$-invariant irreducible character $\varphi$ inducing $\psi$. Now, $\varphi_{K \cap P} \in \operatorname{Irr}_{A\langle g\rangle}(K \cap P)$ induces $\phi$. The subgroup $K \cap P$ is $A\langle g\rangle$-invariant and contains $Z(P)$. However, it is not hard to show that the unique non-trivial $A\langle g\rangle$-invariant subgroups of $P$ containing $Z(P)$ are $\langle x, y, Z(P)\rangle$ and $\langle u, v, Z(P)\rangle$. Now, since $\phi(1)=25$ and $P$ is extraspecial, $\phi_{Z(P)} \neq 1_{Z(P)}$ and this implies that $\phi$ is induced from a character of degree 5 of $K \cap P$. This is a contradiction. 


\section{Characters of Prime power Degree}

Our next aim is to prove Theorem B. In the proof we shall use Isaacs factorisation theory. (See, for instance, [7, Section 21] for an introduction to this subject.) We are going to prove a more general result, from which Theorem $\mathrm{B}$ is an immediate consequence.

TheOREM 3.1. Let $A$ act coprimely on a metanilpotent group $G$. Let $\chi \in$ $\operatorname{Irr}_{A}(G)$. Suppose that $G$ has a nilpotent Hall $\pi$-subgroup, where $\pi$ is the set of primes dividing $\chi(1)$. Then the degrees of any two A-primitive characters inducing $\chi$ coincide.

Proof: We argue by induction on $|G|$. Let $U, V \leqslant G$ be $A$-invariant subgroups of $G$ and $\alpha \in \operatorname{Irr}(U), \beta \in \operatorname{Irr}(V) A$-primitive characters such that $\alpha^{G}=\chi=\beta^{G}$. We want to prove that $\alpha(1)=\beta(1)$. Write $G=K H$ where $H$ is an $A$-invariant $\pi$-complement of $G$ and $K$ is an $A$-invariant Hall $\pi$-subgroup.

Since $\alpha$ and $\beta$ are $A$-primitive characters we know that $\alpha$ and $\beta$ are $\pi$-factorable, that is, $\alpha=\alpha_{\pi} \alpha_{\pi^{\prime}}$ and $\beta=\beta_{\pi} \beta_{\pi^{\prime}}$ where $\alpha_{\pi}$ and $\beta_{\pi}$ are $\pi$-special characters and $\alpha_{\pi^{\prime}}$ and $\beta_{\pi^{\prime}}$ are $\pi^{\prime}$-special (by [5, Lemma 2.6]). Note that, since $\chi(1)$ is a $\pi$-number, $\alpha_{\pi^{\prime}}$ and $\beta_{\pi^{\prime}}$ are linear. Hence, we want to prove that $\alpha_{\pi}(1)=\beta_{\pi}(1)$.

Write $X=K F(G)=K F_{\pi^{\prime}}$ where $F_{\pi^{\prime}}$ is the Hall $\pi^{\prime}$-subgroup of $F(G)$. Of course, since $|G: U|$ and $|G: V|$ are $\pi$-numbers, $F_{\pi^{\prime}}$ is contained in $U \cap V$. Since $G / F(G)$ is nilpotent, $X$ is normal in $G$. Put $U_{1}=U \cap X$ and $V_{1}=V \cap X$. By the uniqueness of Gajendragadkar's factorisation [7, Theorem 21.6], $\alpha_{\pi^{\prime}}$ and $\beta_{\pi^{\prime}}$ are $A$-invariant. Thus, we have that $\left(\alpha_{\pi^{\prime}}\right)_{F_{\pi^{\prime}}}$ and $\left(\beta_{\pi^{\prime}}\right)_{F_{\pi^{\prime}}}$ are $A$-invariant irreducible constituents of $\chi_{F_{\pi^{\prime}}}$. Then by Glauberman's lemma we know that they are conjugate by an element $c \in C_{G}(A)$. Hence, replacing $(V, \beta)$ by $\left(V^{c}, \beta^{c}\right)$, we may assume that $\lambda=\left(\alpha_{\pi^{\prime}}\right)_{F_{\pi^{\prime}}}=\left(\beta_{\pi^{\prime}}\right)_{F_{\pi^{\prime}}}$. Let $T=I_{G}(\lambda)$ be the inertia group of $\lambda$ in $G$. Note that $U$ and $V$ are subgroups of $T$. Suppose that $T<G$. We have that $\alpha^{T}$ and $\beta^{T}$ are Clifford correspondents of $\chi$. Since the Clifford correspondence is a bijection [3, Theorem 6.11], $\alpha^{T}=\beta^{T}$. By the inductive hypothesis it follows that $\alpha(1)=\beta(1)$, as desired. Therefore we may assume that $\lambda$ is $G$-invariant. Let $\hat{\lambda}$ be the canonical extension of $\lambda$ to $X$. By Glauberman's lemma, we may assume that $H$ is contained in $U \cap V$. We have that $\chi_{X} \in \operatorname{Irr}(X)$ (because $(\chi(1),|G: X|)=1$ ). Then

$$
\left(\alpha^{G}\right)_{X}=\left(\alpha_{U_{1}}\right)^{X}=\left(\left(\alpha_{\pi}\right)_{U_{1}}(\widehat{\lambda})_{U_{1}}\right)^{X}
$$

and similarly $\left(\beta^{G}\right)_{X}=\left(\left(\beta_{\pi}\right)_{V_{1}}(\widehat{\lambda})_{V_{1}}\right)^{X}$. Hence,

$$
\left(\left(\alpha_{\pi}\right)_{U_{1}}\right)^{X} \hat{\lambda}=\left(\left(\beta_{\pi}\right)_{V_{1}}\right)^{X} \hat{\lambda}
$$

We deduce that $\left(\left(\alpha_{\pi}\right)_{U_{1}}\right)^{X}=\left(\left(\beta_{\pi}\right)_{V_{1}}\right)^{X}$. Since $F_{\pi^{\prime}}$ is contained in the kernel of this character (see [2, Corollary 5.3]) we may view it as a character of the nilpotent group $X / F_{\pi^{\prime}}$. Now: $\left(\alpha_{\pi}\right)_{U_{1}}$ and $\left(\beta_{\pi}\right)_{V_{1}}$ are $A H / F_{\pi^{\prime}}$-primitive (because $\alpha_{\pi}$ and $\beta_{\pi}$ are $A$-primitive) and induce the same $A H / F_{\pi^{\prime}}$-invariant irreducible character of $X / F_{\pi^{\prime}}$. By Theorem 1.1, $\alpha_{\pi}(1)=\beta_{\pi}(1)$, as desired. 


\section{Proof of Theorem C}

Theorem 3.1 is a generalisation of the theorem of Isaacs, Lewis and Navarro. In this section we prove Theorem $\mathrm{C}$, which is another generalisation. As in the nilpotent case, the key of the proof of Theorem $\mathrm{C}$ is the following lemma.

Lemma 4.1. Let $A$ act coprimely on a solvable group $G$ and let $\chi \in \operatorname{Irr}_{A}(G)$ be A-primitive. Assume that there exists a normal subgroup $N$ of $G$ such that $G / N$ is nilpotent and $N$ has Abelian Sylow subgroups. Then $\chi_{H}$ is homogeneous for every $A$-invariant subgroup $H$ of $G$.

Proof: We may take $N$ to be the nilpotent residual, and thus $N$ is $A$-invariant. We argue by induction on $|G|$. If $\chi$ is not faithful, working on $G / \operatorname{Ker} \chi$ and applying the inductive hypothesis, we have that the restriction of $\chi$ to $H \operatorname{Ker} \chi$ is homogeneous and we deduce that $\chi_{H}$ is homogeneous. Hence, we may assume that $\chi$ is faithful.

Since $G$ has an $A$-primitive faithful irreducible character, we deduce that every $A$ invariant normal Abelian subgroup of $G$ is central (by [3, Corollary 6.13]). Since $N$ has Abelian Sylow subgroups, $F(N)$ is central in $G$. Hence $F(N)=N$ and $G$ is nilpotent. Now we are done by [4, Corollary 2.2].

Lemma 4.2: Suppose that $A$ acts coprimely on a group $G$ and that whenever $D \leqslant U \leqslant G$ are $A$-invariant subgroups and $\alpha$ is an $A$-primitive irreducible character of $U$ then $\alpha_{U}$ is homogeneus. In this situation, if $\alpha$ and $\beta$ are any two $A$-primitive characters of $A$-invariant subgroups of $G$, and $\alpha$ and $\beta$ induce the same irreducible character of $G$, then $\alpha(1)=\beta(1)$.

Proof: It is exactly the same as in [4, Theorem 2.5].

Now we are ready to prove Theorem $\mathrm{C}$, which we restate.

TheOREM 4.3. Let $A$ act coprimely on a solvable group $G$ and let $\chi \in \operatorname{Irr}_{A}(G)$. Assume that there exists a normal subgroup $N$ of $G$ such that $G / N$ is nilpotent and $N$ has Abelian Sylow subgroups. Then the degrees of any two A-primitive irreducible characters inducing $\chi$ coincide.

Proof: It is enough to apply the previous lemma. The hypothesis about the existence and properties of the subgroup $N$ are inherited by all subgroups of $G$, and thus by Lemma 4.1, we see that the hypotheses of Lemma 4.2 hold.

\section{REFERENCES}

[1] E.C. Dade, 'Monomial characters and normal subgroups', Math. Z. 178 (1981), 401-420.

[2] I.M. Isaacs, 'Characters of $\pi$-separable groups', J. Algebra 86 (1984), 98-128.

[3] I.M. Isaacs, Character theory of finite groups (New York, Dover, 1994).

[4] I.M. Isaacs, M.L. Lewis and G. Navarro, 'Invariant characters and coprime action on finite nilpotent groups', Arch. Math. 74 (2000), 401-409. 
[5] I.M. Isaacs and G. Navarro, 'Character correspondences and irreducible induction and restriction', J. Algebra 140 (1991), 131-140.

[6] M.L. Lewis, 'Inducing characters of prime power degree', Osaka J. Math. 37 (2000), 735-744.

[7] O. Manz, T.R. Wolf, Representations of solvable groups, London Math. Society Lecture Notes Series 185 (Cambridge University Press, Cambridge, 1993).

Departamento de Matemáticas

Facultad de Ciencias

Universidad del País Vasco

48080 Bilbao

Spain

e-mail: mtbmoqua@lg.ehu.es
Departament d'Àlgebra

Universitat de València

46100 Burjassot

València

Spain

e-mail: lucia.sanus@uv.es 